\title{
MicroRNA expression profiles in liver and colon of sexually immature gilts after exposure to Fusarium mycotoxins
}

\author{
P. Brzuzan', M. Woźny ${ }^{1}$, L. Wolińska-Nizioł ${ }^{1}$, A. Piasecka ${ }^{1}$, M. Florczyk ${ }^{1}$, \\ E. Jakimiuk², M. Góra ${ }^{3}$, M.K. Luczyński', M. Gajęcki ${ }^{2}$
}

${ }^{1}$ Department of Environmental Biotechnology, Faculty of Environmental Sciences, University of Warmia and Mazury in Olsztyn, Słoneczna 45G, 10-709 Olsztyn, Poland

${ }^{2}$ Department of Veterinary Prevention and Feed Hygiene, Faculty of Veterinary Medicine, University of Warmia and Mazury in Olsztyn, Oczapowskiego 13, 10-950 Olsztyn, Poland

${ }^{3}$ Department of Organic Chemistry, Faculty of Chemistry, Jagiellonian University, Ingardena 3, 30-060 Cracow, Poland

${ }^{4}$ Department of Chemistry, Faculty of Environmental Management and Agriculture, University of Warmia and Mazury in Olsztyn, Pl. Łódzki 4, 10-957 Olsztyn, Poland

\begin{abstract}
To improve our knowledge of the role of microRNAs (miRs) in responses of the porcine digestive system to two Fusarium mycotoxins, zearalenone (ZEN) and deoxynivalenol (DON), we examined the expression of 7 miRs (miR-9, miR-15a, miR-21, miR-34a, miR-122, miR-125b, and miR-192), previously found to be deregulated in diseased liver and colon cells. In this study, immature gilts were exposed to NOEL doses of ZEN (40 $\mu \mathrm{g} / \mathrm{kg} / \mathrm{d})$, DON (12 $\mu \mathrm{g} / \mathrm{kg} / \mathrm{d}), \mathrm{ZEN}+\mathrm{DON}(40+12$ $\mu \mathrm{g} / \mathrm{kg} / \mathrm{d}$ ), and placebo (negative control group) for 7, 14, 21, 28, 35, and 42 days. Before the treatment, expression levels of the selected miRs were measured in the liver, the duodenum, the jejunum, and the ascending and the descending colon of the gilts. Hierarchical clustering of the tissues by their miR expression profiles was consistent with what would be expected based on the anatomical locations and the physiological functions of the organs, suggesting that functions of the miRs are related to the specificities of the tissues in which they are expressed. A subset of 2 pairs of miRs (miR-21+miR-192 and miR-15a+miR-34a), which were assigned to two distinct clusters based on their tissue abundance, was then evaluated in the liver and the ascending and the descending colon during the treatment. The most meaningful results were obtained from the ascending colon, where a significant effect of the treatment was observed, suggesting that during the exposure to mycotoxins, the pathways involved in cell proliferation and survival were disordered. Changes in miR expression in the liver and the descending colon of the treated gilts were smaller, and were associated more with treatment duration than the exposure to ZEN, DON, or ZEN+DON. Further research should focus on identification of genes whose expression is regulated by these aberrantly expressed miRs. This should facilitate understanding of the miRNA-regulated biological effects of mycotoxins.
\end{abstract}

Key words: deoxynivalenol, domestic pig, miRNA expression, zearalenone 


\section{Introduction}

Mycotoxins are biologically active secondary fungal metabolites found as contaminants of feedstuffs which exert toxic effects in animals and human beings (Fink-Gremmels 1999). Fusarium toxins, such as zearalenone (ZEN) and deoxynivalenol (DON), contaminate wheat, maize, and barley worldwide and cause a variety of toxic effects in experimental animals and livestock.

ZEN is a biologically potent, but hardly toxic compound. The most widely acknowledged feature of $\mathrm{ZEN}$ is its ability to bind to estrogen receptor (ER) and induce expression of estrogen responsive genes (e.g. Olsen et al. 2005, Takemura et al. 2007). Consistent with this potential, exposure to $\mathrm{ZEN}$ gives rise to a number of reproductive disorders in mammals, e.g. decreased libido, anovulation, infertility, or neoplasmic lesions (Tiemann et al. 2003, Zinedine et al. 2007, Jakimiuk et al. 2009). Among various mycotoxins found in crops used for food and feed production, DON (also known as vomitoxin), a Fusarium toxin belonging to the trichothecene group, is one of the most prevalent and hazardous (Pinton et al. 2012). The toxicity of DON relies on its ability to cross biological barriers (i.e. intestinal and blood-brain barriers) and to affect the functions and viability of the cells forming such organ systems. The toxin produces a variety of adverse health effects in farm animals, such as inhibition of protein synthesis, reduction of feed intake, and alteration of the immune system (Pestka et al. 2004).

The above facts suggest that adverse effects of ZEN or DON in exposed animals result from multi-pathway processes that are regulated at different levels. Indeed, several studies have identified molecules involved in the signal-transduction and toxicity pathways of the toxins, demonstrating both the complexities and commonalities of their interactions with animal cells. For example, Ahamed et al. (2001) demonstrated that ZEN could stimulate the growth of the ER-positive human breast carcinoma cell line MCF-7 via a MAP kinases signaling cascade. In addition, the toxin functions as an anti-apoptotic agent by increasing the survival of MCF-7 cell cultures undergoing apoptosis caused by serum withdrawal. On the other hand, the ribotoxic DON was found capable of inducing competing apoptotic (p38/p53/Bax/mitochondria/ caspase-3) and survival (ERK/AKT/p90Rsk/Bad) signaling pathways in macrophages (Zhou et al. 2005). Because of their mode of action, microRNAs (miRNAs or miRs), members of the RNA interference (RNA $i$ ) system, seem particularly suitable for studying the molecular processes underlying pathological alterations of cells and tissues at the gene and protein levels (Hudder and Novak 2008).
miRs form a class of endogenously expressed, small, 22 nucleotide long, single-stranded RNAs that bind to the recognition sequences on 3'-untranslated regions (3'-UTRs) of mRNAs and inhibit the expression of specific mRNA targets (Meister and Tuschl 2004). In metazoans, miRNA complementarity to their targets is far from perfect, so one miRNA can bind up to 200 targets, and each mRNA could have recognition sites for more than one miRNA (Betel et al. 2008). The function of miRNAs has been linked to different biological processes, e.g. development and growth control, cell differentiation, signal transduction, cancer, neuronal disease, virus-induced immune defense, programmed cell death, insulin secretion and sensitivity, and metabolism (Ambros 2004). miRs present tremendous therapeutic potential (Garzon et al. 2010).

Some evidence indicates that miRs which regulate pathways and orchestrate integrated responses in intact cells and tissues, also play roles in coordinating networks in toxin-stressed organs, such as the liver (Bala et al. 2009) and the colon (Wu et al. 2008). Environmental or feed contaminants, such as ZEN (Hassen et al. 2007) or DON (Kouadio et al. 2005) which have the potential to induce oxidative stress and hypoxia in animal cells are plausible agents deregulating miRNA expression. Consequently, the aim of this study was to analyze the capability of the Fusarium toxins to affect expression of selected miRNAs in the liver and the colon of immature gilts during the 42 days of exposure to NOEL doses of ZEN, DON, and ZEN+DON mixture. From miRNA studies on pig or mammalian cells we chose miRNAs which play regulatory roles in pathways of signal transduction (miR-9, Rotkrua et al. 2011), apoptosis and cell cycle (miR-15a, Cimmino et al. 2005; miR-21, Chan et al. 2005; miR-34a, Tarasov et al. 2007; miR-125b, Surdziel et al. 2011; miR-192, Ye et al. 2012), fatty-acid metabolism (miR-122, which is a liver specific miRNA, Girard et al. 2008).

\section{Materials and Methods}

\section{Treatment study and sample collection}

The animals were handled in accordance with the regulations set forth by the Local Ethical Commission No. 88/2009 issued on $16^{\text {th }}$ of December 2009. Pigs (Sus scrofa) were purchased from local breeders and kept for 7 days under standard conditions for acclimation. In order to exclude infectious factors that could influence the results concerning indexes of non-specific humoral immunity in the examined animals, serological testing was carried out for Auyeski's disease (PRV), mycoplasmosis (MPS), parvovirosis 
Table 1. Details of Real-Time qPCR primers for selected miRNA targets of this study.

\begin{tabular}{lllll}
\hline \multicolumn{1}{c}{ miRNA } & \multicolumn{1}{c}{ Putative biological process* } & Primer sequence $\left[5^{\prime} \rightarrow 3^{\prime}\right]$ & $\begin{array}{c}\text { Length } \\
{[\mathrm{bp}]}\end{array}$ & miRBase Acc. No. \\
\hline Ssc-miR-9 & signal transduction & tctttggttatctagctgtatg & 22 & MIMAT0025358 \\
\hline Ssc-miR-15a & apoptosis, cell cycle & tagcagcacataatggtttgt & 21 & MIMAT0007753 \\
\hline Ssc-miR-21 & apoptosis, cell cycle & tagcttatcagactgatgttga & 22 & MIMAT0002165 \\
\hline Ssc-miR-34a & cell cycle, signal transduction & tggcagtgtcttagctggttgt & 22 & MIMAT0007757 \\
\hline Ssc-miR-122 & $\begin{array}{l}\text { fatty-acid metabolism, maintenance } \\
\text { of adult liver phenotype }\end{array}$ & tggagtgtgacaatggtgtttgt & 23 & MIMAT0002119 \\
\hline Ssc-miR-125b & cell cycle, signal transduction & tccctgagacctaacttgtga & 22 & MIMAT0002120 \\
\hline Ssc-miR-192 & cell cycle, signal transduction & ctgacctatgaattgacagcc & 21 & MIMAT0013910 \\
\hline
\end{tabular}

* based on literature review; see text for details.

(PPV), actinobacillosis (APP) and porcine reproductive-respiratory syndrome (PRRS). During the acclimation and the further exposure period, all pigs were fed with "blank" (dry) feed that was tested for the presence of background contamination, and found to be free of mycotoxins (ZEN and DON, but also $\alpha$-zearalenol, aflatoxin, and ochratoxin). Throughout the study, the animals had constant access to water and were kept indoors (units with concrete floor and runoff slats, partially covered with insulating rubber mats).

For the treatment study, 75 clinically healthy immature gilts with an initial body weight of about $20 \mathrm{~kg}$ were randomly divided into experimental groups, which were administered per os with NOEL (no observable effect level) doses of ZEN $(40 \mu \mathrm{g} / \mathrm{kg} / \mathrm{d})$, DON $(12 \mu \mathrm{g} / \mathrm{kg} / \mathrm{d})$, DON+ZEN $(12+40 \mu \mathrm{g} / \mathrm{kg} / \mathrm{d})$, or placebo (negative control), for 7, 14, 21, 28, 35, and 42 days. For the exposure, the tested compounds were administered to the treated groups daily with their first (morning) feed. An additional control group consisted of individuals $(n=3)$ which were taken at the beginning of the experiment (control $0 \mathrm{~d}$ ) and were not subjected to any treatment. After each exposure period, randomly selected individuals $(n=3)$ from each experimental group were euthanized and exsanguinated. Immediately after cardiac arrest, fragments of the liver, the duodenum, the jejunum, and the ascending and descending colon were collected and stored in RNAlater ${ }^{\circledR}$ solution (Sigma-Aldrich; Germany) according to the manufacturer's recommendations.

\section{Quantification of miRNAs}

RNA extraction and preparation procedures for qPCR analysis of miRNAs as well as quality check analyses of resulting amplicons followed those described in the paper of Brzuzan et al. (2012). For the purpose of miRNA quantification, quantitative cycle $(\mathrm{Cq})$ values obtained from Real-Time qPCR assay were converted into miRNA copy numbers using a standard curve plot $(\mathrm{Cq}$ versus log copy number $)$ according to the concept of Spachmo and Arukwe (2012).

\section{Hierarchical clustering of similarly expressed miRs}

To investigate a constitutive expression pattern of the miRs (Table 1), their levels were measured at the beginning of the experiment in organs of different physiological condition: the liver, the duodenum, the jejunum, the ascending and the descending colon. For this purpose, $\mathrm{Cq}$ for each assay was transformed into $\Delta \mathrm{Cq}$ against the average $\mathrm{Cq}$ of all assays examined and clustered without centering the data. Normalized data from the assays were subjected to unsupervised hierarchical clustering using Ward's algorithm clustering method and the Euclidean similarity metric and correlation similarity metric to cluster miRNAs and tissues, respectively, without centering the data. All calculations were performed using professional software for gene expression analysis, the GenEx 5 Professional software (MultiD Analyses AB; Sweden).

\section{Statistics}

The correlation of expression of the miRs in groups which were yielded by hierarchical clustering were analyzed using the Pearson method. Statistical differences among constitutive levels of miRNA in gilt organs were tested using analysis of variance (ANOVA) followed by Tukey-Kramer's post hoc multiple comparison test, and before the analysis, 

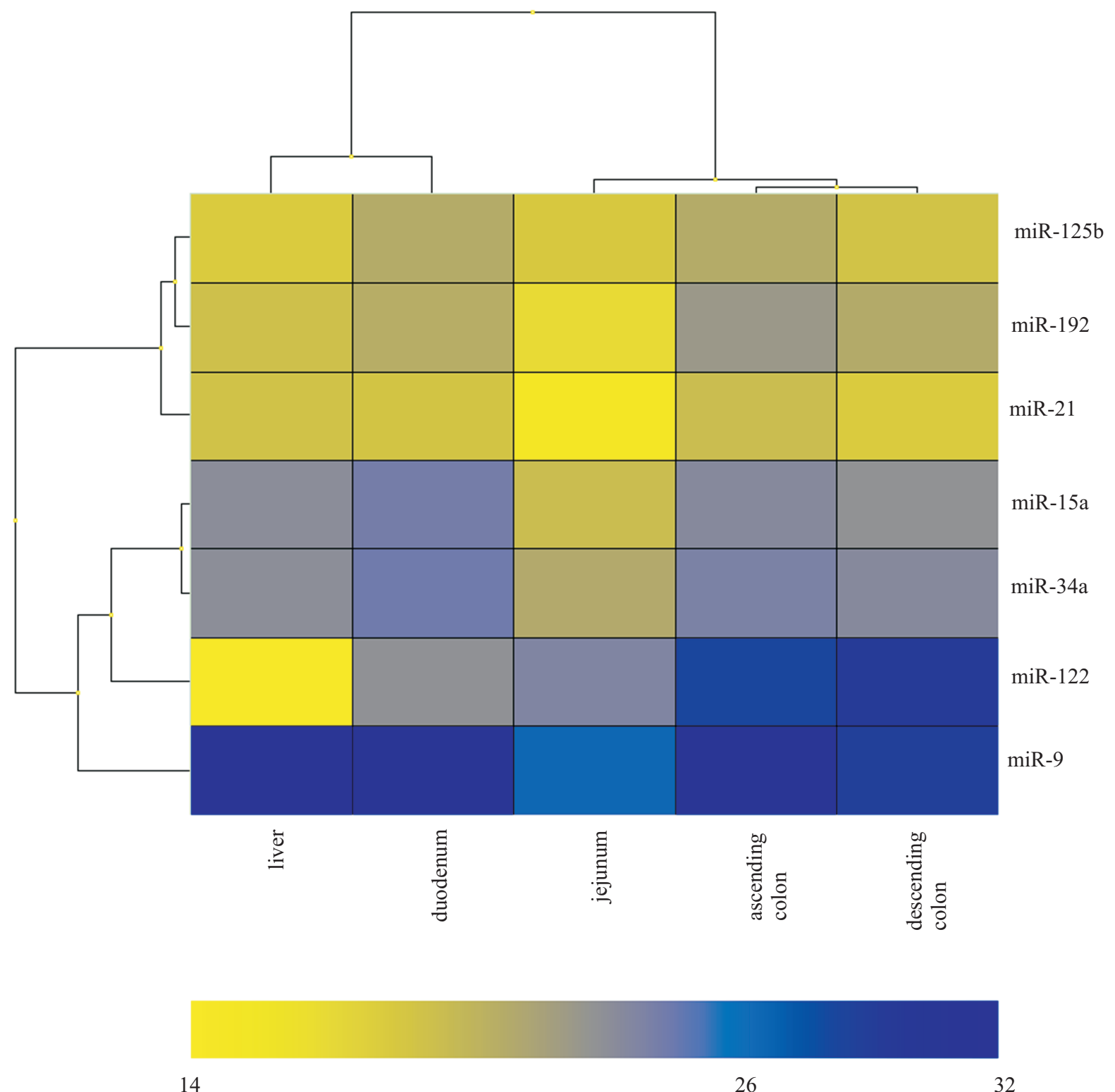

Fig. 1. Unsupervised hierarchical clustering of five immature gilt organs based on the variation of miRNA expression that correlates with the anatomical locations and physiological functions of the tissues. $\mathrm{Cq}$ for each assay was transformed into $\Delta \mathrm{Cq}$ against the average $\mathrm{Cq}$ of all assays examined and clustered without centering the data. A pseudocolor scale outlines the $\mathrm{Cq}$ values represented in the heat map. A view of the clustering pattern of the tissues and the miRs is above and on the right, respectively.

the values were converted to logarithmic scale. One-way ANOVA, followed by Dunnett's post hoc test, were used to compare the means of each of three treatment groups (ZEN, DON, or ZEN+DON) to that in the control (placebo) group from a respective time of treatment. To test if there was a significant difference in the group effect, exposure time or if interactions between these two effects occurred, two-way ANOVA was used to compare the means of the different groups. All calculations and statistical analyses were performed using GenEx 5 Professional software (MultiD Analyses).

\section{Results}

\section{Constitutive expression of miRs in tissues of immature gilts}

Real-Time PCR efficiencies for amplicons of miR targets ranged from 95 to $99 \%$ (coefficient of determination, $R^{2}=0.99$ ), and among the performed runs no appreciable target detection ( $\mathrm{Cq}>40)$ occurred only with the negative controls. Of the 7 miRNAs examined, only miR-9 was found to be expressed at low levels $(\mathrm{Cq}>30)$. PCR product quality analysis showed 
Table 2. Summary of two-way ANOVA results (GenEx 5 Professional) for miRNA expression in organs of gilts from experimental groups.

\begin{tabular}{ccccc}
\hline \multirow{2}{*}{ miRNA } & Source & \multicolumn{3}{c}{ P-value $(P)$} \\
\cline { 3 - 5 } & & liver & ascending colon & descending colon \\
\hline miR-15a & treatment & 0.196 & $<0.001^{*}$ & 0.540 \\
& timepoint & 0.042 & 0.013 & $<0.001^{*}$ \\
& interaction & $<0.001^{*}$ & 0.108 & 0.030 \\
\hline miR-21 & treatment & 0.424 & $<0.001^{*}$ & 0.593 \\
& timepoint & 0.451 & 0.025 & $<0.001^{*}$ \\
& interaction & 0.095 & 0.021 & 0.030 \\
\hline miR-34a & treatment & 0.737 & $0.004^{*}$ & 0.888 \\
& timepoint & $0.001^{*}$ & $0.003^{*}$ & $<0.001^{*}$ \\
& interaction & 0.156 & 0.143 & 0.188 \\
\hline miR-192 & treatment & 0.130 & $<0.001^{*}$ & 0.737 \\
& timepoint & $0.005^{*}$ & 0.017 & 0.050 \\
& interaction & $0.012^{*}$ & $0.002^{*}$ & 0.124 \\
\hline
\end{tabular}

* because of multiple testing a threshold for statistical significance was set at $P<0.013$.

that for the examined miRNA the resulting melting curves had a single peak at about $75^{\circ} \mathrm{C}$, confirming the specificity of the reaction.

To identify the tissue-specific miR profiles, an unsupervised hierarchical clustering based on the variation of expression for each of $7 \mathrm{miRs}$ across the tissues was employed (Fig. 1). The analysis yielded two clusters. In general, the gilt tissues derived from similar anatomical locations and with related physiological functions were primarily clustered together (Fig. 1). For example, tissues derived from the ascending colon were clustered in one group together with the descending colon (pairwise Pearson correlation coefficient, $r=0.971 ; P<0.001)$ and then with the jejunum $(r=0.967 ; P<0.001)$. In the other group, expression of the 7 examined miRs in the liver correlated with that of the duodenum $(r=0.827 ; P=0.022)$.

Our study revealed also 2 differentially expressed miR groups in the studied gilt tissues (Fig. 1). The cluster pattern shown in Figure 1 was confirmed by high correlation coefficients of miRs in either group. The first group of clustered miRNAs that contained miR-125b, miR-192 and miR-21 (Fig. 1) showed the highest expression in the examined organs. miRs from this cluster were significantly more abundant in the liver (except for miR-122) and the duodenum than the remainder miRs in the respective tissues. Pairwise Pearson correlation coefficients for the three miRs ranged between $r=0.542$ (miR-21 and miR-125b) and $r=0.860$ (miR-21 and miR-192), and were not significant. The other cluster of the remaining miRNAs (miR-15a, miR-34a, miR-122 and miR-9) showed a more differentiated expression pattern. For example, miR-122 was found to predominate in the liver, but was only modestly expressed in the remain- ing tissues. Two other miRs, miR-15a and miR-34a, were similarly expressed in low quantities in all tissues $(r=0.981 ; P<0.001)$.

\section{Profiles of miRs in the liver and the colon of gilts treated with Fusarium mycotoxins}

Multiple comparisons of miRNA expression levels between the experimental groups of gilts revealed treatment- and the timepoint-dependent changes in respective organs, as well as an interaction between these two factors (two-way ANOVA; Table 2). Significant effect of the treatment on all examined miRNA expression was observed only in the ascending colon, whereas distinct temporal $\mathrm{miR}$ responses (for miR-15a, miR-21, and miR-34a) were observed in the descending colon (Table 2). In the liver, timepoint-dependent expression changes were noted for two miR species (miR-34a, $P=0.001$; miR-192, $P=0.005$ ). In addition, an interactive effect of treatment and exposure period (timepoint) on miRNA changes for miR-15a and miR-192 was found in tissue of the ascending colon (Table 2).

Figure 2 presents the miRNA expression profile in the liver and the ascending and the descending colon of gilts exposed to NOEL doses of ZEA, DON, and ZEA+DON for 7, 14, 21, 28, 35, and 42 days. In the liver, changes in miRNA where modest with only a few significant $P$ values (Fig. 2A). In comparison to the control group (Dunnett's post hoc) the 21 days of treatment with DON decreased miR-15a level ( $\mathrm{R}=0.5, P<0.01)$, and challenge to ZEA for 35 days resulted in significant up-regulation of miR-15a $(\mathrm{R}=1.6, \quad P<0.01), \quad \mathrm{miR}-21 \quad(\mathrm{R}=1.1, \quad P<0.05)$, and 

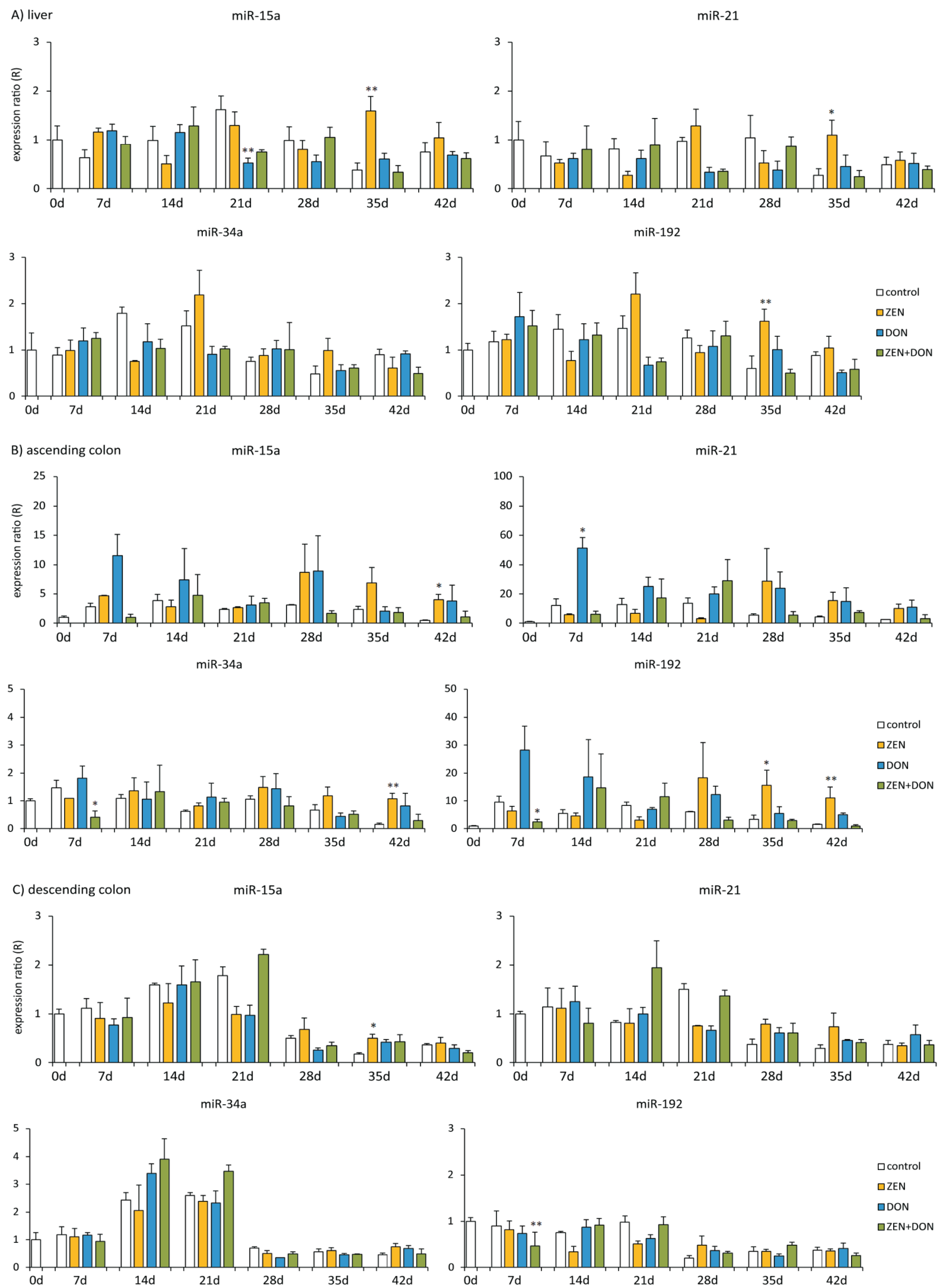

Fig. 2. Changes in miR expression in A) the liver, B) the ascending colon, and C) the descending colon of gilts exposed to ZEA $(40 \mu \mathrm{g} / \mathrm{kg} / \mathrm{d})$, DON $(12 \mu \mathrm{g} / \mathrm{kg} / \mathrm{d})$, and ZEA+DON $(40+12 \mu \mathrm{g} / \mathrm{kg} / \mathrm{d})$ for $7,14,21,28,35$, and 42 days. The bars represent mean values $(n=3)$ of expression ratios $(\mathrm{R} \pm$ S.E. $)$, relative to a control $0 \mathrm{~d}$ group $(\mathrm{R}=1.00)$ at each respective time of the experiment. Data were analyzed using one-way ANOVA, followed by Dunnett's test (GenEx 5 Professional software; MultiD Analyses AB; Sweden). Asterisks indicate groups which showed significantly higher levels of each miRNA over control group at respective timepoint $\left({ }^{*}, P<0.05 ;{ }^{* *}, P<0.01\right)$. 
miR-192 ( $\mathrm{R}=1.6, P<0.01)$. The most meaningful results were obtained from the ascending colon, where significant miR expression changes were observed in each of the exposed groups of the respective timepoint (Fig. 2B). In pigs treated with DON, miRNA expression tended to up-regulate in the first exposure period, while ZEA, on the other hand, seemed to affect the miRNA level at the end of the treatment. For example, 7 days of exposure to DON resulted in a significant 50-fold up-regulation of miR-21 $(\mathrm{R}=51.3$, $P<0.05)$, whereas treatment with ZEA for 42 days increased miRNA levels of miR-15a $(\mathrm{R}=4.0, P<0.05)$, miR-34a $(\mathrm{R}=1.1, P<0.01)$, and miR-192 $(\mathrm{R}=11.0, P<$ 0.01 ) when compared to respective controls (Fig. 2B). Changes in miR expression in the descending colon of the treated gilts were much smaller than those in the ascending colon (Fig. 2C). Noticeably, expression of miR-192 was significantly down-regulated in pigs exposed to ZEA+DON for 7 days, and treatment with ZEA for 35 days caused a significant increase of miR-15a level. Apart from the few significant changes, miR-15a and miR-34a tended to increase after 14 and 21 days of the experiment irrespective of the group (Fig. 2C).

\section{Discussion}

\section{Constitutive expression of miRNAs in gilts}

Quantifying miRNAs in different tissues is an important initial step in investigating their biological functions. To this end, the expression of 7 porcine miRNAs was quantitated in tissues from the control gilts' digestive system, including specimens derived from the liver, the duodenum, the jejunum, and the ascending and the descending colon. We used an unsupervised hierarchical clustering based on the variation of expression for each miRNA across the specimens examined to explore the correlation between tissues taken from different organs. The miRs examined in the study exhibited a wide range of expression levels which were stable and consistent among different parts of the digestive system. In general, normal gilt tissues derived from similar anatomical locations or with related physiological functions were primarily clustered together (Fig. 1). For example, tissues derived from the gastrointestinal system (the colon, and the jejunum), were clustered together, as shown in Fig. 1 . This result recapitulated the previously published clustering patterns of normal mammalian tissues using both miRNA and mRNA expression profiles (Liang et al. 2007). A somewhat surprising clustering of the duodenum with the liver is probably a result of using a limited number of miRs, which affected the sensitivity and specificity of the clustering analysis. Nonetheless, the uniqueness of the tissue clustering using the miR expression profiles may reflect the roles played by those miRNAs in the cellular functioning of different parts of the digestive system in immature gilts.

The cluster analysis yielded two distinct miR groups in the studied gilt tissues (Fig. 1). Although the actual expression values of miRs can vary by orders of magnitude between pigs and humans, their relative abundance in a particular tissue should tend to be more conserved in evolution. Indeed, a general pattern of the orders of individual miRNA amounts in human tissues seems to hold in gilts as well and maintains the following similarities: first, the prominent expression of miR-122 in the liver of immature gilts and low level of this miRNA in other tissues studied (Fig. 1) is consistent with previous data from diverse vertebrate animals (Ason et al. 2006); in humans miR-122 has been proved to have an influence on liver processes such as fatty acid biosynthesis and metabolism, and steroid and steroid hormone biosynthesis (Tsai et al. 2012). Secondly, the high expression of miR-21 in tissues from the digestive system in the gilt corresponds well with the respective levels of normal human tissues (Landgraf et al. 2007). The evolutionarily conserved high expression profile of miR-21 has suggested the important housekeeping cellular roles it plays in differentiation and carcinogenesis (Fujita et al. 2008), and anti-apoptosis (Chan et al. 2005). Furthermore, a relatively high constitutive expression of both miR-125b and miR-192 is consistent with the estimated high copy numbers of these miRNAs in the tissues of human jejunum, duodenum, and proximal and distal colon (Landgraf et al. 2007). Finally, two other miRs, miR-15a and miR-34a, which play a tumor suppressive role in various types of cells (Cimmino et al. 2005, Asslaber et al. 2010), showed consistently low expression levels in all 5 pig organs examined (Fig. 2).

\section{Spatial and temporal changes in miR expression during treatment with Fusarium toxins}

The subset of 2 pairs of miRs (miR-21+miR-192 and miR-15a+miR-34a), which were assigned to two distinct clusters based on their tissue abundance (Fig. 1), was then evaluated in the liver and the ascending and the descending colon during the treatment. The most meaningful results were obtained from the ascending colon, where a significant effect of the treatment was observed, suggesting that during the exposure to mycotoxins, the pathways involved in 
cell proliferation and survival were disordered. Notably, three miRs implicated in limiting cell proliferation and triggering apoptosis in mammalian cells, miR-15a, mir-34a and miR-192 were found to be over-expressed in the ascending colon in the last periods of ZEN exposure (Fig. 2). Together, the aberrant expression of miR-15a, which is a direct transcriptional target of E2F1, a critical downstream target of the tumor suppressor retinoblastoma (Ofir et al. 2011), and elevated levels of miR-34a and miR-192, which mediate cell cycle arrest and apoptosis along the p53 axis (Georges et al. 2008, Asslaber et al. 2010), suggest that various molecular pathways are involved in the response of the gilt cells to chronic exposure to ZEA, and may contribute to its toxicity.

In this study, miRNA expression in the ascending colon of gilts exposed to DON tended to up-regulate in the first exposure periods with significant 50 -fold up-regulation of miR-21 after 7 days of the treatment (Fig. 3). miR-21 is involved in many biological scenarios such as wound healing (Roy and Sen 2011) and breast cancer (Chan et al. 2005). In pigs, predicted mRNA targets include among others programmed cell death protein 4 (PDCD4) or ineterleukin 10 (IL10) that further regulate a set of genes critical to cell proliferation and inflammation (Podolska et al. 2012). Interestingly, in many cell types miR-21 expression pattern is correlated with ERK-MAPK activity (Mei et al. 2013), the intracellular signaling pathways inducing a variety of downstream processes. It was found recently, that DON, besides its effects on cell differentiation and proliferation, may affect the intestinal barrier function through MAPK and claudin proteins (Pinton et al. 2009). Although mechanisms underlying the impairment of barrier functions have been poorly investigated so far, and changes in the miR expression may be the result of a diseased state of the cells, it is tempting to speculate that the likely loss of the barrier function of pig intestinal cells upon treatment with DON is mediated by miR-21. Further research should focus on identification of genes whose expression is regulated by the aberrantly expressed miR, as they might become excellent biomarkers of DON toxicity.

In contrast to apparent modulation of miR expression in the ascending colon of gilts exposed to Fusarium toxins, changes in the liver of the treated gilts were modest, while the effects in the descending colon were associated more with the treatment duration than the exposure to ZEA, DON or mixtures of these mycotoxins (Table 2). One of the explanations for such modest effects of studied mycotoxins on miRNA expression in these tissues could be larger inter-individual variation in miR expression than that in the ascending colon. However, the result may also suggest differential mechanisms of action between the tissues, that differ in cell proliferation status, anatomical location (descending colon) and physiological functions (liver). This argues that more miRNAs should be included in future studies involving experimental groups of larger (i.e. $n>3$ ) sample sizes, to elucidate the role of miRs in the ZEA and DON toxicity mechanism. Consequently, the next generation sequencing methods should be a method of choice to generate more complete lists of miR numbers for determination of spatio-temporal expression changes in healthy and challenged animals.

Spatial and temporal expression patterns determined here for miR-15a, miR-21, miR-34a, and miR-192 in the digestive system of immature pig females challenged by Fusarium toxins are a valuable resource for investigators interested in post-transcriptional gene regulation in the pig and in related mammals, including humans. Further identification of upstream regulators and target genes for the aberrantly expressed miRNAs, performed at early hours of exposure, as well as studies on context-dependent availability of auxilliary factors required for miR function (Glorian et al. 2011) should point to organ specific roles in responses of the digestive tract of immature gilts to mycotoxin exposure.

\section{Acknowledgements}

The research was funded by the Polish Ministry of Science and Higher Education (MNiSW), project No. N R12 008010.

\section{References}

Ahamed S, Foster JS, Bukovsky A, Wimalasena J (2001) Signal transduction through the Ras/Erk pathway is essential for the mycoestrogen zearalenone-induced cell-cycle progression in MCF-7 cells. Mol Carcinog 30: 88-98.

Ambros V (2004) The functions of animal microRNAs. Nature 431: 350-355.

Ason B, Darnell DK, Wittbrodt B, Berezikov E, Kloosterman WP, Wittbrodt J, Antin PB, Plasterk RH (2006) Differences in vertebrate microRNA expression. Proc Natl Acad Sci USA 103: 14385-14389.

Asslaber D, Piñón JD, Seyfried I, Desch P, Stöcher M, Tinhofer I, Egle A, Merkel O, Greil R (2010) microRNA-34a expression correlates with MDM2 SNP309 polymorphism and treatment-free survival in chronic lymphocytic leukemia. Blood 115: 4191-4197.

Bala S, Marcos M, Szabo G (2009) Emerging role of microRNAs in liver diseases. World J Gastroenterol 15: 5633 -5640 .

Betel D, Wilson M, Gabow A, Marks DS, Sander C (2008) The microRNA.org resource: targets and expression. Nucleic Acids Res 36: 149-153. 
Brzuzan P, Woźny M, Wolińska L, Piasecka A (2012) Expression profiling in vivo demonstrates rapid changes in liver microRNA levels of whitefish (Coregonus lavaretus) following microcystin-LR exposure. Aquat Toxicol 122-123: 188-196.

Chan JA, Krichevsky AM, Kosik KS (2005) MicroRNA-21 is an antiapoptotic factor in human glioblastoma cells. Cancer Res 65: 6029-6033.

Cimmino A, Calin GA, Fabbri M, Iorio MV, Ferracin M, Shimizu M, Wojcik SE, Aqeilan RI, Zupo S, Dono M, Rassenti L, Alder H, Volinia S, Liu CG, Kipps TJ, Negrini $\mathrm{M}$, Croce CM (2005) miR-15 and miR-16 induce apoptosis by targeting BCL2. Proc Natl Acad Sci USA 102: 13944-13949.

Fink-Gremmels J (1999) Mycotoxins: their implications for human and animal health. Vet Q 21: 115-120.

Fujita S, Ito T, Mizutani T, Minoguchi S, Yamamichi N, Sakurai K, Iba H (2008) miR-21 gene expression triggered by AP-1 is sustained through a double-negative feedback mechanism. J Mol Biol 378: 492-504.

Garzon R, Marcucci G, Croce CM (2010) Targeting microRNAs in cancer: rationale strategies and challenges. Nat Rev Drug Discov 9: 774-789.

Georges SA, Biery MC, Kim SY, Schelter JM, Guo J, Chang AN, Jackson AL, Carleton MO, Linsley PS, Cleary MA, Chau BN (2008) Coordinated regulation of cell cycle transcripts by p53-inducible microRNAs, miR-192 and miR-215. Cancer Res 68: 10105-10112.

Girard M, Jacquemin E, Munnich A, Lyonnet S, Henrion-Caude A (2008) miR-122, a paradigm for the role of microRNAs in the liver. J Hepatol 48: 648-656.

Glorian V, Maillot G, Poles S, Iacovoni JS, Favre G, Vagner $S$ (2011) HuR-dependent loading of miRNA RISC to the mRNA encodnig the Ras-related small GTP-ase RhoB controls its translation during UV-induced apoptosis. Cell Death Differ 18: 1692-1701.

Hassen W, Ayed-Boussema I, Oscoz AA, Lopez AC, Bacha $\mathrm{H}$ (2007) The role of oxidative stress in zearalenone-mediated toxicity in Hep G2 cells: Oxidative DNA damage, gluthatione depletion and stress proteins induction. Toxicology 232: 294-302.

Hudder A, Novak RF (2008) miRNAs: Effectors of environmental influences on gene expression and disease. Toxicol Sci 103: 228-240.

Jakimiuk E, Gajęcka M, Jana B, Brzuzan P, Zielonka Ł, Skorska-Wyszyńska E, Gajęcki M (2009) Factors determining sensitivity of prepubertal gilts to hormonal influence of zearalenone. Pol J Vet Sci 12: 149-158.

Kouadio JH, Mobioa TA, Baudrimont I, Moukha S, Dano SD, Creppy EE (2005) Comparative study of cytotoxicity and oxidative stress induced by deoxynivalenol, zearalenone or fumonisin B1 in human intestinal cell line Caco-2. Toxicology 213: 56-65.

Landgraf P, Rusu M, Sheridan R, Sewer A, Iovino N, Aravin A, Pfeffer S, Rice A, Kamphorst AO, Landthaler M, Lin C, Socci ND, Hermida L, Fulci V, Chiaretti S, Foà R, Schliwka J, Fuchs U, Novosel A, Muller RU, Schermer B, Bissels U, Inman J, Phan Q, Chien M, Weir DB, Choksi R, De Vita G, Frezzetti D, Trompeter HI, Hornung V, Teng G, Hartmann G, Palkovits M, Di Lauro R, Wernet P, Macino G, Rogler CE, Nagle JW, Ju J, Papavasiliou FN, Benzing T, Lichter P, Tam W, Brownstein MJ, Bosio A, Borkhardt A, Russo JJ, Sander C, Zavolan M, Tuschl
$\mathrm{T}$ (2007) A mammalian microRNA expression atlas based on small RNA library sequencing. Cell 129: 1401-1414.

Liang Y, Ridzon D, Wong L, Chen C (2007) Characterization of microRNA expression profiles in normal human tissues. BMC Genomics 8: 166.

Mei Y, Bian C, Li J, Du Z, Zhou H, Yang Z, Zhao RC (2013) miR-21 modulates the ERK-MAPK signaling pathway by regulating SPRY2 expression during human mesenchymal stem cell differentiation. J Cell Biochem 114: 1374-1384.

Meister G, Tuschl T (2004) Mechanisms of gene silencing by double-stranded RNA. Nature 431: 343-349.

Ofir M, Hacohen D, Ginsberg D (2011) MiR-15 and miR-16 are direct transcriptional targets of E2F1 that limit E2F-induced proliferation by targeting cyclin E. Mol Cancer Res 9: 440-447.

Olsen CM, Meussen-Elholm ET, Hongslo JK, Stenersen J, Tollefsen KE (2005) Estrogenic effects of environmental chemicals: an interspecies comparison. Comp Biochem Physiol C Toxicol Pharmacol 141: 267-274.

Pestka JJ, Zhou HR, Moon Y, Chung YJ (2004) Cellular and molecular mechanisms for immune modulation by deoxynivalenol and other trichothecenes: unraveling a paradox. Toxicol Lett 153: 61-73.

Pinton P, Nougayrède JP, Del Rio JC, Moreno C, Marin DE, Ferrier L, Bracarense AP, Kolf-Clauw M, Oswald IP (2009) The food contaminant deoxynivalenol decreases intestinal barrier permeability and reduces claudin expression. Toxicol Appl Pharmacol 237: 41-48.

Pinton P, Guzylack-Piriou L, Kolf-Clauw M, Oswald IP (2012) The effect on the intestine of some fungal toxins: the trichothecenes. Curr Immunol Rev 8: 193-208.

Podolska A, Anthon C, Bak M, Tommerup N, Skovgaard K, Heegaard PM, Gorodkin J, Cirera S, Fredholm M (2012) Profiling microRNAs in lung tissue from pigs infected with Actinobacillus pleuropneumoniae. BMC Genomics 13: 459.

Rotkrua P, Akiyama Y, Hashimoto Y, Otsubo T, Yuasa Y (2011) MiR-9 downregulates CDX2 expression in gastric cancer cells. Int J Cancer 129: 2611-2620.

Roy S, Sen CK (2011) MiRNA in innate immune responses: novel players in wound inflammation. Physiol Genomics 43: 557-565.

Spachmo B, Arukwe A (2012) Endocrine and developmental effects in Atlantic salmon (Salmo salar) exposed to perfluorooctane sulfonic or perfluorooctane carboxylic acids. Aquat Toxicol 108: 112-124.

Surdziel E, Cabanski M, Dallmann I, Lyszkiewicz M, Krueger A, Ganser A, Scherr M, Eder M (2011) Enforced expression of miR-125b affects myelopoiesis by targeting multiple signaling pathways. Blood 117: 4338-4348.

Takemura H, Shim JY, Sayama K, Tsubura A, Zhu BT, Shimoi K (2007) Characterization of the estrogenic activities of zearalenone and zeranol in vivo and in vitro. J Steroid Biochem Mol Biol 103: 170-177.

Tarasov V, Jung P, Verdoodt B, Lodygin D, Epanchintsev A, Menssen A, Meister G, Hermeking H (2007) Differential regulation of microRNAs by 533 revealed by massively parallel sequencing: $m i R-34 a$ is a p53 target that induces apoptosis and G1-arrest. Cell Cycle 6: 1586-1593.

Tiemann U, Tomek W, Schneider F, Vanselow J (2003) Effects of the mycotoxins alpha- and beta-zearalenol on regulation of progesterone synthesis in cultured 
granulosa cells from porcine ovaries. Reprod Toxicol 17: 673-681.

Tsai WC, Hsu SD, Hsu CS, Lai TC, Chen SJ, Shen R, Huang Y, Chen HC, Lee CH, Tsai TF, Hsu MT, Wu JC, Huang HD, Shiao MS, Hsiao M, Tsou AP (2012) MicroRNA-122 plays a critical role in liver homeostasis and hepatocarcinogenesis. J Clin Invest 122(8): 2884-2897.

Wu F, Zikusoka M, Trindade A, Dassopoulos T, Harris ML, Bayless TM, Brant SR, Chakravarti S, Kwon JH (2008) MicroRNAs are differentially expressed in ulcerative colitis and alter expression of macrophage inflammatory peptide-2 alpha. Gastroenterology 135: 1624-1635.
Ye L, Su X, Wu Z, Zheng X, Wang J, Zi C, Zhu G, Wu S, Bao W (2012) Analysis of Differential miRNA Expression in the Duodenum of Escherichia coli F18-Sensitive and -Resistant Weaned Piglets. PLoS ONE 7: e43741.

Zhou HR, Jia Q, Pestka JJ (2005) Ribotoxic stress response to the trichothecene deoxynivalenol in the macrophage involves the SRC family kinase Hck. Toxicol Sci 85: 916-926.

Zinedine A, Soriano JM, Moltó JC, Mañes J (2007) Review on the toxicity, occurrence, metabolism, detoxification, regulations and intake of zearalenone: an oestrogenic mycotoxin. Food Chem Toxicol 45: 1-18. 